\title{
INFRARED MOLECULAR SPECTROSCOPY OF ORION
}

\author{
NEAL J. EVANS II \\ Astronomy Department, \\ The University of Texas, Austin Texas 78712, USA \\ E-mail NJE@ASTRO.AS.UTEXAS.EDU
}

\begin{abstract}
Recent results on infrared spectroscopy of the cluster of sources in the Orion molecular cloud are discussed. These results imply very high abundances of $\mathrm{CO}, \mathrm{C}_{2} \mathrm{H}_{2}$, $\mathrm{HCN}$, and OCS, suggesting that grain surface chemistry has been important.
\end{abstract}

Keywords : infrared spectroscopy, interstellar molecules, molecular clouds

\section{Introduction}

This review will consider new results on the infrared cluster in the Orion molecular cloud. This region of massive star formation has a rich chemistry (see Genzel \& Stutzki 1989 for a review). There seem to be several different regions with distinctive kinematics and chemical abundances. Of particular interest to this meeting are the hot core, where evidence suggests that molecules have recently evaporated from grain mantles (Blake et al. 1987), and the plateau feature, thought to be an outflow from IRc2, which has enhanced emission from sulfur compounds. Because of space limitations in this article, I will restrict myself to infrared absorption spectroscopy of interstellar molecules, leaving discussion of emission from atoms, ions, or $\mathrm{H}_{2}$ to other reviewers.

Infrared spectroscopy of rovibrational lines of interstellar molecules allows one to determine the populations of many rotational levels with observations at very similar wavelengths. Since infrared lines can be observed in absorption against embedded infrared sources, one can obtain an effective resolution equal to the size of the infrared source, although one is limited to observations along the lines of sight to such sources. Comparison of infrared absorption lines and millimeter emission lines can provide valuable information on geometry and kinematics, since only the gas in front of the source is seen in absorption in the infrared. Determination of column densities from absorption studies does not depend on intensity calibration, since the column density is determined from the equivalent width, a quantity unaffected by calibration errors. Finally, one can detect symmetric molecules such as $\mathrm{C}_{2} \mathrm{H}_{2}$ (Lacy et al. 1989a) and $\mathrm{CH}_{4}$ (Lacy et al. 1991), which have no permanent dipole moment, rendering them undetectable by rotational spectroscopy.

On the negative side of the ledger, most of the vibrational bands lie at $\lambda$ between 3 and $15 \mu \mathrm{m}$, where atmospheric attenuation allows observations in only a few windows. The best of these windows, around $10 \mu \mathrm{m}$, is also compromised by interstellar attenuation by the silicate feature. The first of these problems could be solved by high-resolution spectrometers $\left(\lambda / \Delta \lambda \gtrsim 1 \times 10^{4}\right)$ on large space telescopes, 
but none of the currently planned space missions have the right characteristics to exploit this opportunity.

\section{The Observations}

\section{1. THE SOURCES}

The dense molecular cloud behind the Orion Nebula contains a complex group of infrared sources. Extinction has a major effect on the appearance of the region even at fairly long infrared wavelengths (see the maps in Wynn-Williams et al. 1984). The most prominent source at $\lambda \lesssim 20 \mu \mathrm{m}$ is the Becklin-Neugebauer Object (BN), while IRc2, suspected to be the most luminous embedded source, is most apparent in the maps at 7.8 and $12.5 \mu \mathrm{m}$, local minima in the interstellar extinction (Draine and Lee 1983). Recent images of this region at $12.4 \mu \mathrm{m}$ (Gezari et al. 1991) show IRc2, IRc7, and IRc4 to be strong and distinct sources, with IRc4 clearly extended and complex. Emission at all $\lambda \lesssim 30 \mu \mathrm{m}$ shows a distinct drop to the south of IRc2 and east of IRc4, a fact explained by extremely high extinction associated with the hot core component, as mapped by $\mathrm{NH}_{3}(\mathrm{~J}, \mathrm{~K})=(3,2)$ emission (see Genzel and Stutzki 1989).

\section{2. THE BECKLIN-NEUGEBAUER OBJECT}

The pioneering work in infrared absorption spectroscopy of molecules in starforming regions was the study of CO toward BN (Scoville et al. 1983). In addition to band-head emission in the overtone bands near $2.3 \mu \mathrm{m}$, they observed discrete absorption features in the fundamental band near $4.6 \mu \mathrm{m}$ at velocities of $-18,-3$, and $+9 \mathrm{~km} \mathrm{~s}^{-1}$. The last velocity component was naturally attributed to the ambient cloud (or the ridge component), while the blue-shifted features were assumed to be material in an outflow. Both the -18 and $+9 \mathrm{~km} \mathrm{~s}^{-1}$ features were fitted by a single temperature of $150 \mathrm{~K}$. More recent work on $\mathrm{CO}$ absorption toward other sources is reviewed by Maillard in this volume.

\section{3. IRC2, IRC7, AND IRC4}

Because of the heavy extinction to these sources, absorption spectroscopy has been difficult until high-resolution spectrometers were developed for longer wavelengths (Lacy et al. 1989b). Using a cryogenic echelle with a velocity resolution of about $20 \mathrm{~km} \mathrm{~s}^{-1}$, Lacy et al. (1989a) were able to detect $\mathrm{C}_{2} \mathrm{H}_{2}$ toward IRc2, using the $\nu_{5}$ vibrational band near $13.5 \mu \mathrm{m}$. More recently, $\mathrm{CH}_{4}$ has also been detected, using the $\nu_{4}$ band at about $7.6 \mu \mathrm{m}$, toward IRc2, as well as toward several other sources (Lacy et al. 1991). These were the first detections of these molecules in interstellar clouds.

A map of $\mathrm{C}_{2} \mathrm{H}_{2} \mathrm{R}(5)$ equivalent width (Evans, Lacy, \& Carr 1991) shows a strong peak on IRc2, with more extended absorption encompassing IRc7 and IRc4, but increasing to the southeast, in the direction of the hot core, suggesting that both the hot core and the plateau feature (centered on IRc2) may have enhanced 
$\mathrm{C}_{2} \mathrm{H}_{2}$ abundances. In addition to the heavily blended $\mathrm{Q}$ branch, a total of 5 wellseparated, unresolved $R$ branch lines have been seen toward IRc2, with an average velocity of $-3 \mathrm{~km} \mathrm{~s}^{-1}$, suggesting that they arise in the approaching edge of the low velocity outflow centered on IRc2. Analysis of these lines indicates that the lower J lines are quite saturated and thus narrow (inferred FWHM linewidth of 2.7 $\mathrm{km} \mathrm{s}^{-1}$ ). The saturation is confirmed by the detection of several $\mathrm{R}$ branch lines and the Q branch of ${ }^{13} \mathrm{CCH}_{2}$. After correcting for this saturation, Evans et al. (1991) derive a temperature of about $150 \mathrm{~K}$ for the gas in the $\mathrm{C}_{2} \mathrm{H}_{2}$ absorbing region.

A second band of $\mathrm{C}_{2} \mathrm{H}_{2}$, the combination band $\nu_{4}+\nu_{5}$, was also detected during the study of $\mathrm{CH}_{4}$ at $7.6 \mu \mathrm{m}$. Analysis of five lines in this weaker band toward IRc2 again indicates some saturation. The correction which gives the best fit to a single temperature results in $T=140 \mathrm{~K}$ and about three times higher column density of $\mathrm{C}_{2} \mathrm{H}_{2}$ than was derived from the $\nu_{5}$ lines. Compared to the lines in the $\nu_{5}$ band these lines are less blueshifted (average velocity of $+7 \mathrm{~km} \mathrm{~s}^{-1}$ ) and wider (FWHM $\Delta v=5.8 \mathrm{~km} \mathrm{~s}^{-1}$ ). These differences may be understood in the context of an accelerating flow centered on IRc2. Because the extinction is considerably less at $7.6 \mu \mathrm{m}$ than at $13.5 \mu \mathrm{m}$, absorption can be seen from closer to the source at $7.6 \mu \mathrm{m}$. This picture would explain the larger column density, while the velocity differences could be explained if the flow is accelerating in the region probed at 7.6 $\mu \mathrm{m}$, but not in the region probed at $13.5 \mu \mathrm{m}$.

In the $\mathrm{C}_{2} \mathrm{H}_{2}$ spectra toward IRc2, there are also lines of the $\nu_{2}$ band of $\mathrm{HCN}$. In all, five lines of $\mathrm{HCN}$ have been detected from levels up to $J=17$. The average velocity of these lines is $0.8 \mathrm{~km} \mathrm{~s}^{-1}$. The populations of the levels up to $J=14$ are consistent with a thermal distribution, while the population of $J=17$ is clearly sub-thermal. Indirect evidence, based on the strength of a blend of the $\mathrm{C}_{2} \mathrm{H}_{2} \mathrm{R}(3)$ line with the $\mathrm{HCN} \mathrm{R}(8)$ line, indicates that the $\mathrm{HCN}$ lines are also narrow and somewhat saturated. Consequently, the equivalent widths were corrected for saturation, assuming the same intrinsic width as the $\mathrm{C}_{2} \mathrm{H}_{2}$ lines. The resulting temperature, based on $J$ up to 14 , is about $150 \mathrm{~K}$, consistent with the temperature determined from $\mathrm{C}_{2} \mathrm{H}_{2}$. Models of excitation, using a large-velocity-gradient code, indicate that densities of $3 \times 10^{6} \mathrm{~cm}^{-3}$ to $1 \times 10^{7} \mathrm{~cm}^{-3}$ can account for the thermalization of levels up to $J=14$ while also explaining the sub-thermal population of the $J=17$ level.

Bands of OCS $\left(\nu_{1}\right), \mathrm{CO},{ }^{13} \mathrm{CO}$, and probably $\mathrm{C}^{18} \mathrm{O}$ were detected toward IRc2 in spectra centered around $4.9 \mu \mathrm{m}$. Overlapping lines complicated the analysis, but with 13 lines detected, OCS is clearly present. Levels up to $J=32$ in OCS appear to be thermalized at $T \sim 140 \mathrm{~K}$. Based on analysis of two ${ }^{13} \mathrm{CO}$ lines and an assumed isotope ratio of 50 , the $\mathrm{CO}$ column density is $1.5 \times 10^{20} \mathrm{~cm}^{-2}$, but this number is very uncertain, partly because the ${ }^{13} \mathrm{CO}$ lines have $\mathrm{P}-\mathrm{Cygni}$ profiles.

\section{Abundances and Implications for Astrochemistry}

Using estimates of the extinction to $\mathrm{BN}$ or IRc2 and a conversion from $A_{V}$ to $N_{\mathrm{H}_{2}}$, one can estimate abundances of $\mathrm{CO}$ and (for IRc2 only) the other species. 
For $\mathrm{CO}$, the resulting abundance is quite high $\left(\geq 5 \times 10^{-4}\right)$, implying that all the carbon must be in gaseous $\mathrm{CO}$ toward both BN and IRc2 (though the estimate for $N(C O)$ in the latter source is quite uncertain). This conclusion may imply that $N_{H_{2}}$ has been underestimated or that $\mathrm{C}>\mathrm{O}$ in this region. Additional support for a carbon-rich chemistry comes from the high abundance of $\mathrm{HCN}\left(5 \times 10^{-7}\right.$ to $5 \times 10^{-6}$ ) toward IRc2, which can be matched by models with $\mathrm{C}>\mathrm{O}$ (e.g., Langer and Graedel 1989). The $\mathrm{C}_{2} \mathrm{H}_{2}$ abundance $\left(3 \times 10^{-7}\right.$ to $\left.6 \times 10^{-6}\right)$ is best understood in terms of models where molecules have been frozen on grain mantles, preserving early time abundances to be released when newly-formed stars heat the grains (e.g., Brown, Charnley, \& Millar 1988). Such a model has already been suggested for the hot core (Blake et al. 1987) and may also be relevant for the plateau. Evidence for a more active role for the grains is provided by the observations of a high abundance of solid $\mathrm{CH}_{4}$, which suggests $\mathrm{CH}_{4}$ formation in the grain mantles (Lacy et al. 1991).

Portions of this research were supported by grants from the National Science Foundation and the Texas Advanced Research Program.

\section{References}

Blake, G. A., Sutton, E. C., Masson, C. R., and Phillips, T. G. 1987, Ap. J., 315, 621.

Brown, P. D., Charnley, S. B., and Millar, T. J. 1988, MNRAS, 231, 409.

Draine, B. T., and Lee, H. M. 1984, Ap. J., 285, 89.

Evans, N. J., II, Lacy, J. H., and Carr, J. S. 1991, Ap. J., in press (Dec. 20).

Genzel, R., and Stutzki, J. 1989, ARA $B A, \mathbf{2 7}, 11$.

Gezari, D. 1991, Ap. J., in prep..

Lacy, J. H., Evans, N. J., II, Achtermann, J. M., Bruce, D. E., Arens, J. F., and Carr, J. S. 1989a, Ap. J. (Letters), 342, L43.

Lacy, J. H., Achtermann, J. M., Bruce, D. E., Lester, D. F., Arens, J. F., Peck, M.

C., and Gaalema, S. D. 1989b, Pub. Astr. Soc. Pac., 101, 1166.

Lacy, J. H., Carr, J. S., Evans, N. J., II, Baas, F., Achtermann, J. M., and Arens,

J. F. 1991, $A p$. J., 376, 556.

Langer, W. D., and Graedel, T. E. 1989, Ap. J. Suppl., 69, 241.

Scoville, N. Z., Hall, D. N. B., Kleinmann, S. G., and Ridgway, S. T. 1983, Ap. J., $275,201$.

Wynn-Williams, C. G., Genzel, R., Becklin, E. E., and Downes, D. 1984, Ap. J., 281, 172. 


\section{QUESTIONS AND ANSWERS}

J.P.Maillard: (1) The unique temperature you estimate seems to me not in contradiction with what I reported toward luminous YSOs. In opposite, it confirms the existence of a quiescent warm gas enriched in neutral molecules released from the warm grains. (2) However, the column densities you determine may be criticized by the large difference between the width of line profiles $(<2 \mathrm{~km} / \mathrm{s})$ required for fitting the data and your resolution of $20-25 \mathrm{~km} / \mathrm{s}$. In addition, different widths are required depending on the molecule. How can that be physically possible?

N.Evans: (1) Yes, I agree. Essentially we see only the warm, enhanced abundance region (hot core and/or plateau) and not the ambient cloud (ridge) in Orion. (2) Again, I agree that it is important to improve our resolution and John Lacy is proposing to do this. For the moment, we have to rely on indirect arguments for narrow lines, but I think they are convincing. I would admit that there are uncertainties whenever lines are unresolved, but the column density estimates are still probably more certain that most of those obtained from emission lines, which have problems with unknown beam filling. 